\title{
Relationship between body composition indices and cardiovascular disease risk factors in Iranian inactive female college students
}

\begin{abstract}
Purpose: To determine which is the best anthropometric index among body mass index (BMI), waist to hip ratio (WHR), waist to stature ratio (WSR) and C index in relation to cardiovascular diseases risk factors.

Methods: This cross-sectional study was carried out in a sample of 300 Iranian female college students, aged 18-36 years that were selected via multistage cluster random sampling from university of Fars province. Fasting blood sugar, lipid profile including total cholesterol (TC), Low density lipoprotein cholesterol (LDL), high density lipoprotein cholesterol (HDL) and triglycerides (TG) were estimated by standard enzymatic procedures and anthropometric indices were measured. Pearson correlation coefficients and multiple linear regressions were used in analysis.

Results: The correlation between BMI and all cardiovascular diseases risk factors were statistically significant. There was positive correlation between WHR and WSR with TC, TG and LDL, while there was a negative correlation between WSR and HDL. There was no correlation between $\mathrm{C}$ index and cardiovascular diseases risk factors except for TG. Multiple regression analysis confirmed the predictive power of BMI, WHR and WSR for cardiovascular diseases risk factors and BMI was the best simple anthropometric index in predicting cardiovascular diseases risk factors in non-obese female college students.
\end{abstract}

Conclusion: These data support the hypothesis that BMI, WSR, WHR and C index may be considered as risk factors for cardiovascular disease and BMI is a better index for predicting some cardiovascular risk factors in young non-obese women.

Keywords: body mass index, waist to hip ratio, waist to stature ratio, C index, cardiometabolic risk factor, non-obese women, predictive power

\section{Introduction}

Cardiovascular diseases are the leading cause of mortality and disability throughout the world. ${ }^{1,2}$ About 17 million people die annually as a consequence of cardiovascular diseases. ${ }^{3}$ Prevalence of cardiovascular diseases risk factors is high in Iran. ${ }^{3,4}$ Similar to many other developing countries, rapid lifestyle changes made Iranian prone to cardiovascular diseases and their risk factors. ${ }^{5}$ And in contrast to trends observed in northern Europe and the USA, mortality from cardiovascular diseases is increasing in Iran that may be attributed to a rising prevalence of obesity. ${ }^{6}$

Epidemiological studies have found a progressive increase in the prevalence of cardiovascular risk factors (dyslipidaemia elevated blood pressure, disturbances in glycaemic control) with increasing body fatness. ${ }^{7}$ Although abdominal visceral adipose tissue measured by computed tomography (CT) or magnetic resonance imaging may more accurately reflect body fat distribution to predict metabolic risks, the inherent high cost and radiation hazard prevent their use in largescale epidemiological studies. Therefore, a variety of anthropometric indices have been used as a proxy for total fat and abdominal fat to assess risk for diseases, particularly cardiovascular diseases. ${ }^{8} \mathrm{BMI}$, WHR, WSR, $\mathrm{C}$ index all are found to associate with cardiovascular risk factors. ${ }^{9}$ Body mass index (BMI) is a simple measure of body size and is the most widely used method to estimate the prevalence of obesity in a population. Nevertheless, it is a crude index that does not take into account the distribution of body fat. On the other hand, WC, WHR, WSR and C index are used as a surrogate for body fat
Volume 8 Issue 2 - 2019

\author{
Farzaneh Movaseghi,' Nasibeh Kazemi, ${ }^{2}$ \\ Rezvan Rezvani Asl, ${ }^{3}$ Fatemeh Movaseghi ${ }^{4}$ \\ 'Department of Physical education and Sport Sciences, Sepidan \\ Branch, Islamic Azad University, Iran \\ ${ }^{2}$ Department of Physical education and Sport Sciences, Research \\ and Sciences Branch, Islamic Azad University, Iran \\ ${ }^{3}$ Department of Physical education and Sport Sciences, \\ Marvdasht Branch, Islamic Azad University, Iran \\ ${ }^{4}$ M.S of public health, Shiraz University, Iran
}

\begin{abstract}
Correspondence: Farzaneh Movaseghi, Department of Physical education and Sport Sciences, Sepidan Branch, Islamic Azad University, Sepidan, Fars, Iran, Tel +98-917-1876733,
\end{abstract} Email fmovaseghi24@yahoo.com

Received: July 15, 2018 | Published: April 03, 2019 centralization. ${ }^{10}$ The anthropometric-metabolic risk relationship is influenced by age, gender, and ethnic differences. And predictive power of anthropometric indices is population-dependent and is likely to be different in different ethnic groups. ${ }^{10}$ So the best index of obesity that is predictive for CVD risk remains controversial and such study in younger population especially females is meagre. Furthermore, there are few studies available that explore the accuracy of such measures in normal weight young women in developing countries. The aim of this study was to verify the relation between anthropometric indices and cardiovascular disease risk factors (lipid profile) and to determine which anthropometric index is a better predictor of cardiovascular risk factors in non-obese Iranian female college students.

\section{Materials and methods}

\section{Study subjects}

This cross-sectional study was conducted on Fars province universities. 300 healthy female college students aged 18 to 35 years without any previous systemic diseases or medications related to body weight change or affecting glucose and lipid levels, were included in our study. All the individuals were volunteers and gave their informed consent for participation into the study.

\section{Anthropometric measurements}

Weight was measured while the subjects were minimally clothed, without shoes using digital scales (Seca Model 840, Hanvor) and recorded to the nearest $0.1 \mathrm{~kg}$. Height was measured in a standing 
position without shoes, while the shoulders were in normal position using a wall -mounted digital stadiometer (Seca Model 242, Hanvor). Body mass index was calculated as participants' weight in kilograms divided by the square of height in meters $\left(\mathrm{kg} / \mathrm{m}^{2}\right)$. Waist circumference $(\mathrm{cm})$ was measured at the end of normal expiration, with the measuring tape positioned at the midway between the lower rib and the iliac crest. Hip circumference (HC) was measured at the point yielding maximum circumference over the buttocks. WHR was calculated as WC $(\mathrm{cm})$ divided by hip circumference $(\mathrm{cm})$ and WSR was calculated as $\mathrm{WC}(\mathrm{cm})$ divided by height $(\mathrm{cm})$. C index was constructed using the following formula: $\mathrm{C}$ index $=$ Waist Circumference $(\mathrm{m}) /[0.109 \times \sqrt{ }$ $\{$ Body weight $(\mathrm{kg}) /$ Height $(\mathrm{m})\}$ ]

\section{Blood sampling and analysis}

Blood samples were collected between 7:00 and 8:00 A.M after a 12-hour overnight fast. Analysis of samples was performed using a Selectra 2 auto-analyzer (Vital Scientific, Spankeren, Netherlands). Total cholesterol (TC) and triglycerides (TG) were assayed by enzymatic colorimetric tests with cholesterol esterase, cholesterol oxidase, and glycerol phosphate oxidase (Pars Azmon Kits, Iran). High-density lipoprotein cholesterol (HDL-C) was measured using the same method after precipitation of the apolipoprotein B containing lipoproteins with phosphotungstic acid. Serum glucose concentration was assayed using the enzymatic colorimetric method with the glucose oxidase technique. Low-density lipoprotein cholesterol (LDL-C) was calculated according to the Friedewald equation. ${ }^{11}$

\section{Statistical analysis}

Initially, the descriptive analysis of variables was presented by means of proportions, means and standard deviation. The anthropometric variables were used as predictors and indicators of the cardiovascular diseases risk factors (TC,TG,LDL,HDL,FBS). Pearson correlation coefficients were calculated to investigate the association between anthropometric variables and cardiovascular diseases risk factors. Multiple linear regressions (stepwise method) was carried out to determine the best anthropometric index of obesity that is predictive for CVD risk factors. All statistical analyses were performed by SPSS version 17 (SPSS Inc, Chicago, IL, USA). A p-value $<0.05$ was considered significant.

\section{Results}

Data of 300 female college students were completed and included in the final analysis in this study. The mean age was 24.24 years $( \pm 4.83)$ with a range of 18 to 36 years. Of the 300 participants, 11 were obese (BMI between $30-33.35 \mathrm{~kg} / \mathrm{m}^{2}$ ). The mean (SD) of anthropometric indices and metabolic characteristics of the study population are presented in Table1. Table 2 illustrates the correlation matrix of the variables. Body mass index had significant correlations with the entire serum lipid values. WHR and WSR had significant correlations with all of the serum lipid values except fasting blood sugar .Significant correlation was found just between $\mathrm{C}$ index and TG ( $<<0.05$; Table 2$)$. Table 3 shows the predictive power of BMI, WHR, WSR and C index for cardiovascular diseases risk factors. The value of $\mathrm{R} 2$ showed that BMI is a better predictor for Fasting blood sugar, Total cholesterol and HDL, whereas WSR is a significant predictor for Triglyceride and WHR is a significant predictor for LDL.

Table I Mean and standard deviation of characteristics of the study participants, anthropometric indices and cardiovascular diseases risk factors

\begin{tabular}{lll}
\hline Variables & Mean & SD \\
\hline Age (year) & 24.24 & 4.83 \\
Weight $(\mathrm{kg})$ & 57.45 & 9.94 \\
Height $(\mathrm{cm})$ & 160.022 & 5.82 \\
BMI & 22.41 & 3.55 \\
WHR & 0.75 & 0.05 \\
WSR & 0.45 & 0.05 \\
C Index & 1.11 & 0.065 \\
TC (mg/dL) & 162.24 & 33.49 \\
TG (mg/dL) & 86.39 & 37.24 \\
HDL (mg/dL) & 46.25 & 11.8 \\
LDL (mg/dL) & 97.04 & 27.72 \\
FBS (mg/dL) & 85.82 & 9.26 \\
\hline
\end{tabular}

Abbreviations: BMI, Body mass index (kg/m2); WHR, Waist-to-hip ratio: WSR, Waist-to-stature ratio; TC, Total cholesterol (mg/dL); TG, Triglycerides $(\mathrm{mg} / \mathrm{dL})$; HDL, High-density lipoprotein; LDL, Low-density lipoprotein; FBS, Fasting blood sugar $(\mathrm{mg} / \mathrm{dL})$

Table 2 Pearson correlation coefficients between anthropometric indices and cardiovascular diseases risk factors in non-obese female college student

\begin{tabular}{lllll}
\hline Variables & BMI & WHR & WSR & C Index \\
\hline Total Cholesterol(mg/dL) & $0.150^{*}$ & $0.143^{*}$ & $0.140^{*}$ & 0.079 \\
Triglycerides $(\mathrm{mg} / \mathrm{dL})$ & $0.295^{* * *}$ & $0.215^{* * *}$ & $0.306 * * *$ & $0.169 * *$ \\
HDL Cholesterol(mg/dL) & $-0.17 I^{* *}$ & -0.078 & $-0.166^{* *}$ & -0.093 \\
LDL Cholesterol(mg/dL) & $0.132^{*}$ & $0.138^{*}$ & $0.13 I^{*}$ & 0.083 \\
Fasting Blood Sugar $(\mathrm{mg} / \mathrm{dL})$ & $0.125^{*}$ & -0.033 & 0.071 & -0.011 \\
\hline
\end{tabular}

*Correlation is significant at the 0.05 level (2-tailed), ${ }^{* *}$ Correlation is significant at the 0.0 I level (2-tailed), ${ }^{* * *}$ Correlation is significant at the $0.00 \mathrm{I}$ level ( 2 -tailed). Table 3 Multiple regression analysis of relationship between anthropometric indices and cardiovascular diseases risk factors in non-obese female college students

\begin{tabular}{|c|c|c|c|c|}
\hline Dependent Variable & Explanatory Variables & Independent Variable Coefficients $(\beta)$ & P-Value & R-Square \\
\hline Total Cholesterol(mg/dL) & BMI & 0.15 & 0.015 & 0.019 \\
\hline Triglycerides(mg/dL) & WSR & 0.306 & 0 & 0.1 \\
\hline HDL Cholesterol(mg/dL) & BMI & -0.171 & 0.005 & 0.029 \\
\hline LDL Cholesterol(mg/dL) & WHR & 0.138 & 0.026 & 0.019 \\
\hline Fasting Blood Sugar(mg/dL) & BMI & 0.125 & 0.045 & 0.016 \\
\hline
\end{tabular}




\section{Discussion}

The aim of this study was to evaluate BMI, WHR, WSR and C index as anthropometric indices associated with cardiovascular disease risk factors in non-obese female college students. In this study, we found that some anthropometric indices are significantly associated with cardiovascular diseases risk factors, and of all the anthropometric indices BMI was the best, in predicting these factors in Iranian nonobese female college student. Finding of our study are consistent with Kotian, ${ }^{12}$ Ghorbanian, ${ }^{13}$ and Oliveira ${ }^{1}$ as we also found that BMI was the anthropometric indicator with strongest relation to lipid profile. In contrast Pua \& Ong, ${ }^{14}$ suggested a WSR as the best indicator for CVD risk factors in Singaporean women. Subjects age difference between two studies could, to some extent, explained the different finding in these studies as It has been shown that age modifies the discriminative ability of anthropometric indices to identify subjects with CVD risk factors. ${ }^{15-17}$ Furthermore different studies have given varying conclusions about which anthropometric measure has the best predictive capacity for CVD risk factors and anthropometric indices proposed in Western countries with taller populations might be not applicable in Asian ethnicities, and consequently the necessity of using different anthropometric indices in different populations is obvious .Assessing the risk for the presence of major CVD risk factors in adults, especially young adults, is of particular importance since it allows us to easily identify those at high risk for development of clinical CVD later in life particularly for those with metabolically obese despite a normal weight because they display metabolic disturbance that may predispose them to higher cardiovascular risk factors. Although abdominal obesity is increasingly recognized as a major risk factor for cardiovascular disease and anthropometric measurements of abdominal obesity appear to be more strongly associated with metabolic risk factors, ${ }^{18,19}$ only small amounts of intra-abdominal fat are physiologically present before adulthood. ${ }^{20}$ However, more clinical and nonclinical intensive investigations are needed in other parts of Iran to determine which anthropometric index is the best for predicting cardiovascular diseases risk factors among Iranian young non-obese women. Also our investigation has several limitations that must be acknowledged.

a) These Iranian college students of Fars province, in our sample weren't obese and limits our ability to generalize our results to overweight and obese female college students

b) Because the participants in our study sample were between 18 and 36 years of age, our findings may not be applicable to the older women

c) Our study sample was inactive female college students so we can't generalize our results to active female college student.

\section{Conclusion}

The results of this study showed that anthropometric measurements that provide obesity indices (Body mass index, waist to-hip ratio, waist to-stature ratio and $\mathrm{C}$ index) are useful non-invasive methods of obtaining information on cardiovascular diseases risk factors even in non-obese individual and BMI is a better index for predicting some cardiovascular risk factors in young female college students who are not obese.

\section{Acknowledgments}

None.

\section{Conflicts of interest}

The author declares there are no conflicts of interest.

\section{References}

1. Oliveira MAMD, Fagundes RLM, Moreira EAM, et al.Relationship between anthropometric indicator and risk factors for cardiovascular disease. Arquivos Brasileiros de Cardiologia. 2010;94(4):451-457.

2. Krusa MP, Hallage T, Gama MPR, et al. Programmed Ventricular Pacing in the Management of Patients with Brugada Syndrome. Arquivos Brasileiros de Cardiologia. 2007;89(2):147-153.

3. Miandoabi T, Sadeghi M, S Hemirani H. Is lipoprotein (A)a predictor of coronary artery disease severity? ARYA Atherosclerosis Journal. 2008;4(2):49-52.

4. Esmaillzadeh A, Mirmiran P, zizi F, et al. Waist-to-hip ratio is a better screening measure for cardiovascular risk factors than other anthropometric indicators in Tehranian adult men. International Journal of Obesity and Related Metabolic Disorders. 2004;28(10):1325-1332.

5. Sarrafzadegan N, Kelishadi R, Najafian AR, et al. Anthropometric Indices in Association with Cardiometabolic Risk Factors: Findings of the Isfahan Healthy Heart Program. ARYA Atherosclerosis Journal. 2010;5(4):152-162.

6. Azizi F, Esmaillzadeh A, Mirmiran P. Obesity and cardiovascular disease risk factors in Tehran adults: a population-based study. Eastern Mediterranean Health Journal. 2004;10(6):887-897.

7. Shidfar F, Alborzi F, Salehi M, et al. Association of waist circumference, body mass index and conicity index with cardiovascular risk factors in postmenopausal women. Cardiovascular Journal of Africa. 2012;23(8):442-445

8. Browning LM, Hsieh SD, Ashwell M. A systematic review of waistto-height ratio as a screening tool for the prediction of cardiovascular disease and diabetes: $0 \cdot 5$ could be a suitable global boundary value. Nutrition Research Reviews. 2010;23(2):247-269.

9. Meerjady Sabrina F, CGN Mascie T, Mahmudur R. Conicity Index of adult bangladeshi population and their socio-demographic characteristics. Ibrahim Medical College Journal. 2009;3(1):1-8.

10. Khader YS, Batieha A, Jaddou H, et al. Cutoff values for detecting metabolic abnormalities in Jordanian adults, Diabetes. Metabolic Syndrome and Obesity: Targets and Therapy. 2010;3:395-402.

11. Friedewald WT, Levy RI, Fredrickson DS. Estimation of the concentration of low density lipoprotein cholesterol in plasma without use of the preparative ultracentrifuge. Clinical Chemistry. 1972;18(6):499-502.

12. Kotian GB, Prathapchandra Kedilaya H. BMI is the best anthropometric index to predict cardiovascular disease risks in young adult females. International Journal of Pharmaceutical Sciences Review and Research. 2013;22(1):188-191.

13. Ghorbanian B. Relation between anthropometric indicators and serum lipid profiles as cardiovascular risk factors personals of Iranian Azarbayjan University of Shahid Madani. European Journal of Sports and Exercise Science. 2012;1(3):52-58.

14. Pua YH, Ong PH. Anthropometric indices as screening tools for cardiovascular risk factors in Singaporean women. Asia Pacific Journal of Clinical Nutrition. 2005;14(1):74-79.

15. Neufeld LM, Jones-Smith JC, García R, et al. Anthropometric predictors for risk of chronic disease in non diabetic, non hypertensive young Mexican women. Public Health Nutrition. 2008;11(2):159-167. 
16. Lin WY, Lee LT, Chen CY, et al. Optimal cut off values for obesity using simple anthropometric indices to predict cardiovascular risk factors in Taiwan. International Journal of Obesity and Related Metabolic Disorders. 2002;26(9):1232-1238.

17. Zhu S, Wang Z, Hishka S, et al. Waist circumference and obesity associated risk factors among whites in the third National Health and Nutrition Examination Survey clinical actione thresholds. American Journal of Clinical Nutrition. 2002;76(4):743-749.
18. Wang Y, Rimm EB, Stampfer MJ, et al. Comparison of abdominal adiposity and overall obesity in predicting risk of type 2 diabetes among men. American Journal of Clinical Nutrition. 2005;81(3):555-563.

19. Despres JP, Lemieux I. Abdominal obesity and the metabolic syndrome. Nature. 2006;444(7121):881-887.

20. Freiberg MS, Pencina MJ, D’Agostino RB, et al. BMI vs. waist circumference for identifying vascular risk. Obesity (Silver Spring). 2008;16(2):463-469 